\title{
ON SOME NEW PARANORMED SEQUENCE SPACES OF FUZZY NUMBERS DEFINED BY ORLICZ FUNCTIONS AND STATISTICAL CONVERGENCE
}

\section{A. ESI}

Adiyaman University,Science and Art Faculty, Department of Mathematics

02040, Adiyaman, Turkey

E-mail: aesi23@hotmail.com

Received August 24, 2005; revised September 2, 2006; published online December 15, 2006

\begin{abstract}
In this paper we introduce the concept of strongly $\lambda(p)$ convergence of fuzzy numbers with respect to an Orlicz function and examine some properties of the resulting sequence spaces and $\lambda(p)$ - statistical convergence. It is also shown that if a sequence of fuzzy numbers is strong $\lambda(p)$ convergent with respect to an Orlicz function then it is $\lambda(p)$ - statistically convergent.
\end{abstract}

Key words: Orlicz function,paranorm, de la Vallee-Poussin means, fuzzy numbers

\section{Introduction and Preliminaries}

The concept of paranorm is closely related to linear metric spaces.It is a generalization of an absolute value definition. Let $X$ be a linear space. A function $g: X \rightarrow R$ is called paranorm, if

i) $g(0)=0$,

ii) $g(x) \geq 0$, for all $x \in X$,

iii) $g(-x)=g(x)$, for all $x \in X$,

iv) $g(x+y) \leq g(x)+g(y)$, for all $x, y \in X$,

v) if $\left(\alpha_{n}\right)$ is a sequence of scalars with $\alpha_{n} \rightarrow \alpha(n \rightarrow \infty)$ and $\left\{x_{n}\right\}$ a sequence of vectors with $g\left(x_{n}-x\right) \rightarrow 0(n \rightarrow \infty)$, then $g\left(\alpha_{n} x_{n}-\alpha x\right) \rightarrow 0(n \rightarrow \infty)$.

The last property is called continuity of multiplication by scalars. The space is called the paranormed space $X$ with the paranorm $g$.

A function $M:[0, \infty[\rightarrow[0, \infty[$ is an Orlicz function if it is continuous, non-decreasing and convex with

$$
M(0)=0, M(x)>0 \text { for } x>0, \quad M(x) \rightarrow \infty \text { as } x \rightarrow \infty .
$$


An Orlicz function is said to satisfy $\Delta_{2}$-condition for all values of $u$, if there exists a constant $K>0$, such that

$$
M(2 u) \leq K M(u), \quad u \geq 0 .
$$

Lindenstrauss and Tzafriri [5] used the idea of Orlicz function to construct the sequence space

$$
l_{M}=\left\{x=\left(x_{k}\right): \sum_{k} M\left(\frac{\left|x_{k}\right|}{\rho}\right)<\infty, \text { for some } \rho>0\right\} .
$$

The space $l_{M}$ with the norm

$$
\|x\|=\inf \left\{\rho>0: \sum_{k} M\left(\frac{\left|x_{k}\right|}{\rho}\right) \leq 1\right\}
$$

becomes a Banach space which is called an Orlicz sequence space. The space $l_{M}$ is closely related to the space $l_{p}$ which is an Orlicz sequence space with $M(x)=x^{p}, 1 \leq p<\infty$.

In the later stage different Orlicz sequence spaces were introduced and studied by Parashar and Choudhary [10], Esi, Isik and Esi [4], Esi [2], Esi and Et [3], and many others.

The purpose of this paper is to introduce and study the concepts of $\lambda(p)$ strong convergence of fuzzy numbers with respect to an Orlicz function and $\lambda(p)$-statistical convergence and some relations between them.

Let $p=\left(p_{k}\right) \in l_{\infty}$, then the following well-known inequality will be used in the paper: for sequences $\left(a_{k}\right)$ and $\left(b_{k}\right)$ of complex numbers we have

$$
\left|a_{k}+b_{k}\right|^{p_{k}} \leq K\left(\left|a_{k}\right|^{p_{k}}+\left|b_{k}\right|^{p_{k}}\right)
$$

where $K=\max \left(1,2^{H-1}\right)$ and $H=\sup _{k} p_{k}$.

We now give here a brief introduction about the sequences of fuzzy numbers (see [1] and [12]). Let $D$ denote the set of all bounded intervals $A=[\underline{A}, \bar{A}]$ on the real line $R$. For $A, B \in D$, define

$$
\begin{aligned}
& A \leq B \text { if and only if } \underline{A} \leq \underline{B} \text { and } \bar{A} \leq \bar{B}, \\
& d(A, B)=\max \{|\underline{A}-\underline{B}|,|\bar{A}-\bar{B}|\} .
\end{aligned}
$$

Then it can be easily seen that $d$ defines a metric on $D$ and $(D, d)$ is a complete metric space [1]. Also the relation $\leq$ is a partial order on $D$.

A fuzzy number is a fuzzy subset of the real line $R$ which is bounded, convex and normal. Let $L(R)$ denote the set of all fuzzy numbers which are upper semicontinuous and have compact support, i.e. if $X \in L(R)$ then for any $\alpha \in[0,1], X^{\alpha}$ is compact, where

$$
\begin{aligned}
& X^{\alpha}=\{t: X(t) \geq \alpha, \quad \text { if } \alpha \in(0,1]\} \\
& X^{0}=c l(\{t \in R: X(t)>\alpha, \text { if } \alpha=0\}),
\end{aligned}
$$


where $\operatorname{cl}(A)$ is the closure of $A$. The set $R$ of real numbers can be embedded in $L(R)$ if we define $\bar{r} \in L(R)$ by

$$
\bar{r}(t)= \begin{cases}1, & \text { if } t=r, \\ 0, & \text { if } t \neq r .\end{cases}
$$

The additive identity and multiplicative identity of $L(R)$ are denoted by $\overline{0}$ and $\overline{1}$, respectively. Then the arithmetic operations on $L(R)$ are defined as follows:

$$
\begin{aligned}
& (X \oplus Y)(t)=\sup \{X(s) \wedge Y(t-s)\}, \quad t \in R, \\
& (X \Theta Y)(t)=\sup \{X(s) \wedge Y(s-t)\}, \quad t \in R, \\
& (X \otimes Y)(t)=\sup \{X(s) \wedge Y(t / s)\}, \quad t \in R, \\
& (X / Y)(t)=\sup \{X(s t) \wedge Y(s)\}, \quad t \in R,
\end{aligned}
$$

These operations can be defined in terms of $\alpha$-level sets as follows:

$$
\begin{aligned}
& {[X \oplus Y]^{\alpha}=\left[a_{1}^{\alpha}+b_{1}^{\alpha}, a_{2}^{\alpha}+b_{2}^{\alpha}\right],} \\
& {[X \Theta Y]^{\alpha}=\left[a_{1}^{\alpha}-b_{1}^{\alpha}, a_{2}^{\alpha}-b_{2}^{\alpha}\right],} \\
& {[X \otimes Y]^{\alpha}=\left[\min _{i \in\{1,2\}} a_{i}^{\alpha} b_{i}^{\alpha}, \max _{i \in\{1,2\}} a_{i}^{\alpha} b_{i}^{\alpha}\right],} \\
& {\left[X^{-1}\right]^{\alpha}=\left[\left(a_{2}^{\alpha}\right)^{-1},\left(a_{1}^{\alpha}\right)^{-1}\right], a_{i}^{\alpha}>0}
\end{aligned}
$$

for each $0<\alpha \leq 1$.

For $r \in R$ and $X \in L(R)$, the product $r X$ is defined as follows:

$$
r X(t)= \begin{cases}X\left(r^{-1} t\right), & \text { if } r \neq 0 \\ 0, & \text { if } r=0\end{cases}
$$

Define a map

$$
\bar{d}: L(R) \times L(R) \rightarrow R_{+} \cup\{0\}
$$

by $\bar{d}(X, Y)=\sup _{0 \leq \alpha \leq 1} d\left(X^{\alpha}, Y^{\alpha}\right)$. For $X, Y \in L(R)$ define $X \leq Y$ if and only if $X^{\alpha} \leq Y^{\alpha}$ for any $\alpha \in[0,1]$. It is known that $(L(R), \bar{d})$ is a complete metric space [6].

A metric on $L(R)$ is said to be a translation invariant if

$$
\bar{d}(X+Z, Y+Z)=\bar{d}(X, Y), \text { for } X, Y, Z \in L(R) .
$$

Lemma 1. [7]. If $\bar{d}$ is a translation invariant metric on $L(R)$ then

i) $\bar{d}(X+Y, \overline{0}) \leq \bar{d}(X, \overline{0})+\bar{d}(Y, \overline{0})$,

ii) $\bar{d}(\lambda X, \overline{0}) \leq|\lambda| \bar{d}(X, \overline{0}), \quad|\lambda|>1$. 
A sequence $X=\left(X_{k}\right)$ of fuzzy numbers is a function $X$ from the set $N$ of natural numbers into $L(R)$. The fuzzy number $X_{k}$ denotes the value of the function at $k \in N$.

A sequence $X=\left(X_{k}\right)$ of fuzzy numbers is said to be bounded if the set $\left\{X_{k}: k \in N\right\}$ of fuzzy numbers is bounded.

A sequence $X=\left(X_{k}\right)$ of fuzzy numbers is said to be converge to a fuzzy number $X_{0}$ if for every $\varepsilon>0$ there is a positive integer $N$ such that $\bar{d}\left(X_{k}, X_{0}\right)<\varepsilon$ for $k>N$.

\section{Some New Sequence Spaces}

Recently, Nuray and Savas [9] have defined the following space of fuzzy numbers:

$$
l(p)=\left\{X=\left(X_{k}\right): \sum_{k} \bar{d}\left(X_{k}, \overline{0}\right)^{p_{k}}<\infty\right\},
$$

where $p=\left(p_{k}\right)$ is a bounded sequence of strictly positive real numbers. If $p_{k}=$ $p$ for all $k$, then $l(p)=l_{p}$, the space due to Nanda [8]. Lately, Mursaleen and Basarir [7] have defined the following spaces of sequences of fuzzy numbers:

$$
\begin{aligned}
& F(p)=\left\{X=\left(X_{k}\right): \lim _{n} \frac{1}{n} \sum_{k=1}^{n} \bar{d}\left(X_{k}, X\right)^{p_{k}}=0\right\}, \\
& F_{0}(p)=\left\{X=\left(X_{k}\right): \lim _{n} \frac{1}{n} \sum_{k=1}^{n} \bar{d}\left(X_{k}, \overline{0}\right)^{p_{k}}=0\right\}, \\
& F_{\infty}(p)=\left\{X=\left(X_{k}\right): \sup _{n} \frac{1}{n} \sum_{k=1}^{n} \bar{d}\left(X_{k}, \overline{0}\right)^{p_{k}}<\infty\right\}
\end{aligned}
$$

and called them the spaces of sequences of fuzzy numbers which are strongly convergent to $X_{0}$, strongly convergent to zero and strongly bounded, respectively.

In this paper, we define the following spaces:

Definition 1. Let $\Lambda=\left(\lambda_{n}\right)$ be a non-decreasing sequence of positive real numbers tending to infinity and $\lambda_{1}=1$ and $\lambda_{n+1} \leq \lambda_{n}+1$ and let $M$ be an Orlicz function, $p=\left(p_{k}\right)$ be any sequence of strictly positive real numbers and $X=\left(X_{k}\right)$ be sequence of fuzzy numbers, then for some $\rho$

$$
\begin{aligned}
& F[M, \lambda, p]=\left\{X=\left(X_{k}\right): \lim _{n} \frac{1}{\lambda_{n}} \sum_{k \in I_{n}}\left[M\left(\frac{\bar{d}\left(X_{k}, X_{0}\right)}{\rho}\right)\right]^{p_{k}}=0\right\}, \\
& F_{0}[M, \lambda, p]=\left\{X=\left(X_{k}\right): \lim _{n} \frac{1}{\lambda_{n}} \sum_{k \in I_{n}}\left[M\left(\frac{\bar{d}\left(X_{k}, \overline{0}\right)}{\rho}\right)\right]^{p_{k}}=0\right\},
\end{aligned}
$$




$$
F_{\infty}[M, \lambda, p]=\left\{X=\left(X_{k}\right): \sup _{n} \frac{1}{\lambda_{n}} \sum_{k \in I_{n}}\left[M\left(\frac{\bar{d}\left(X_{k}, \overline{0}\right)}{\rho}\right)\right]^{p_{k}}<\infty\right\}
$$

where $I_{n}=\left[n-\lambda_{n}+1, n\right]$.

We denote $F[M, \lambda, p], F_{0}[M, \lambda, p]$ and $F_{\infty}[M, \lambda, p]$ as $F[M, \lambda], F_{0}[M, \lambda]$ and $F_{\infty}[M, \lambda]$ when $p_{k}=1$ for all $k$. If $X=\left(X_{k}\right) \in F[M, \lambda, p]$, we say that $X=\left(X_{k}\right)$ is strongly $\lambda(p)$-convergent to fuzzy number $X_{0}$ with respect to the Orlicz function $M$. If $M(x)=x$ and $\lambda_{n}=n$ then

$$
F[M, \lambda, p]=F(p), \quad F_{0}[M, \lambda, p]=F_{0}(p), \quad F_{\infty}[M, \lambda, p]=F_{\infty}(p),
$$

which were defined by Mursaleen and Basarir [7].

\section{Main Results}

In this section we examine some topological properties of spaces $F[M, \lambda, p]$, $F_{0}[M, \lambda, p]$ and $F_{\infty}[M, \lambda, p]$. If $d$ is a translation invariant, we have the following theorem.

Theorem 1. For any Orlicz function $M$ and any sequence $p=\left(p_{k}\right)$ of strictly positive real numbers, $F[M, \lambda, p], F_{0}[M, \lambda, p]$ and $F_{\infty}[M, \lambda, p]$ are linear spaces over the set of complex numbers.

Proof. We shall prove the theorem only for $F_{0}[M, \lambda, p]$. The other cases can be treated similarly. Let $X=\left(X_{k}\right), Y=\left(Y_{k}\right) \in F_{0}[M, \lambda, p]$ and $\alpha, \beta \in C$. In order to prove the result we need to find some $\rho_{3}>0$ such that

$$
\lim _{n} \frac{1}{\lambda_{n}} \sum_{k \in I_{n}}\left[M\left(\frac{\bar{d}\left(\alpha X_{k}+\beta Y_{k}, \overline{0}\right)}{\rho_{3}}\right)\right]^{p_{k}}=0 .
$$

Since $X=\left(X_{k}\right), Y=\left(Y_{k}\right) \in F_{0}[M, \lambda, p]$, there exist some $\rho_{1}>0$ and $\rho_{2}>0$ such that

$$
\lim _{n} \frac{1}{\lambda_{n}} \sum_{k \in I_{n}}\left[M\left(\frac{\bar{d}\left(X_{k}, \overline{0}\right)}{\rho_{1}}\right)\right]^{p_{k}}=0, \quad \lim _{n} \frac{1}{\lambda_{n}} \sum_{k \in I_{n}}\left[M\left(\frac{\bar{d}\left(Y_{k}, \overline{0}\right)}{\rho_{2}}\right)\right]^{p_{k}}=0 .
$$

Define $\rho_{3}=\max \left(2|\alpha| \rho_{1}, 2|\beta| \rho_{2}\right)$. Since $M$ is non decreasing and convex, we get

$\frac{1}{\lambda_{n}} \sum_{k \in I_{n}}\left[M\left(\frac{\bar{d}\left(\alpha X_{k}+\beta Y_{k}, \overline{0}\right)}{\rho_{3}}\right)\right]^{p_{k}} \leq \frac{1}{\lambda_{n}} \sum_{k \in I_{n}}\left[M\left(\frac{\bar{d}\left(\alpha X_{k}, \overline{0}\right)}{\rho_{3}}+\frac{\bar{d}\left(\beta Y_{k}, \overline{0}\right)}{\rho_{3}}\right)\right]^{p_{k}}$ 


$$
\begin{aligned}
& \leq \frac{1}{\lambda_{n}} \sum_{k \in I_{n}} \frac{1}{2^{p_{k}}}\left[M\left(\frac{\bar{d}\left(X_{k}, \overline{0}\right)}{\rho_{1}}\right)+M\left(\frac{\bar{d}\left(Y_{k}, \overline{0}\right)}{\rho 2}\right)\right]^{p_{k}} \\
& \leq \frac{1}{\lambda_{n}} \sum_{k \in I_{n}} \frac{1}{2^{p_{k}}}\left[M\left(\frac{\bar{d}\left(X_{k}, \overline{0}\right)}{\rho_{1}}\right)+M\left(\frac{\bar{d}\left(Y_{k}, \overline{0}\right)}{\rho 2}\right)\right]^{p_{k}} \\
& \leq \frac{K}{\lambda_{n}} \sum_{k \in I_{n}}\left[M\left(\frac{\bar{d}\left(X_{k}, \overline{0}\right)}{\rho_{1}}\right)\right]^{p_{k}}+\frac{K}{\lambda_{n}} \sum_{k \in I_{n}}\left[M\left(\frac{\bar{d}\left(Y_{k}, \overline{0}\right)}{\rho_{2}}\right)\right]^{p_{k}} \rightarrow 0 \text { as } n \rightarrow \infty,
\end{aligned}
$$

where $K=\max \left(1,2^{H-1}\right), H=\sup _{k} p_{k}$, so that $\alpha X+\beta Y \in F_{0}[M, \lambda, p]$. This completes the proof.

Theorem 2. For any Orlicz function $M$ and a bounded sequence $p=\left(p_{k}\right)$ of strictly positive real numbers, $F[M, \lambda, p]$ and $F_{0}[M, \lambda, p]$ are paranormed spaces with

$$
g(X)=\inf \left\{\rho^{p_{n} / H}:\left(\frac{1}{\lambda_{n}} \sum_{k \in I_{n}}\left[M\left(\frac{\bar{d}\left(X_{k}, \overline{0}\right)}{\rho_{1}}\right)\right]^{p_{k}}\right)^{1 / M} \leq 1, \quad n \geq 1\right\},
$$

where $M=\max (1, H)$.

Proof. Clearly $g(\overline{0})=0$ and $g(X)=g(-X)$. Since $\bar{d}$ is a translation invariant, it can be seen easily that

$$
g(X+Y) \leq g(X)+g(Y), \text { for } X=\left(X_{k}\right), Y=\left(Y_{k}\right) \in F_{0}[M, \lambda, p] .
$$

Since $M(0)=0$, we get $\inf \left\{\rho^{p_{n} / H}\right\}=0$ for $X=\overline{0}$. Conversely, suppose that $g(X)=0$, then

$$
\inf \left\{\rho^{p_{n} / H}:\left(\frac{1}{\lambda_{n}} \sum_{k \in I_{n}}\left[M\left(\frac{\bar{d}\left(X_{k}, \overline{0}\right)}{\rho}\right)\right]^{p_{k}}\right)^{1 / M} \leq 1, \quad n \geq 1\right\}=0 .
$$

This implies that for a given $\varepsilon>0$, there exists some $\rho_{\varepsilon}\left(0<\rho_{\varepsilon}<\varepsilon\right)$ such that

$$
\left(\frac{1}{\lambda_{n}} \sum_{k \in I_{n}}\left[M\left(\frac{\bar{d}\left(X_{k}, \overline{0}\right)}{\rho_{\varepsilon}}\right)\right]^{p_{k}}\right)^{1 / M} \leq 1 .
$$

Thus for each $n$ we get

$$
\left(\frac{1}{\lambda_{n}} \sum_{k \in I_{n}}\left[M\left(\frac{\bar{d}\left(X_{k}, \overline{0}\right)}{\varepsilon}\right)\right]^{p_{k}}\right)^{1 / M} \leq\left(\frac{1}{\lambda_{n}} \sum_{k \in I_{n}}\left[M\left(\frac{\bar{d}\left(X_{k}, \overline{0}\right)}{\rho_{\varepsilon}}\right)\right]^{p_{k}}\right)^{1 / M} \leq 1 .
$$

Suppose that $\bar{d}\left(X_{n_{m}}, 0\right) \neq 0$ for some $m \in I_{n}$. Let $\varepsilon \rightarrow 0$, then $\left(\frac{\bar{d}\left(X_{n_{m}}, \overline{0}\right)}{\varepsilon}\right) \rightarrow$ $\infty$. It follows that

$$
\left(\frac{1}{\lambda_{n}} \sum_{k \in I_{n}}\left[M\left(\frac{\bar{d}\left(X_{n_{m}}, \overline{0}\right)}{\varepsilon}\right)\right]^{p_{k}}\right)^{1 / M} \rightarrow \infty
$$


which is a contradiction. Therefore $X_{n_{m}} \neq 0$. Finally, we prove that scalar multiplication is continuous. Let $\gamma$ be any complex number. By definition

$$
g(\gamma X)=\inf \left\{\rho^{p_{n} / H}:\left(\frac{1}{\lambda_{n}} \sum_{k \in I_{n}}\left[M\left(\frac{\bar{d}\left(\gamma X_{k}, \overline{0}\right)}{\rho}\right)\right]^{p_{k}}\right)^{1 / M} \leq 1, n \geq 1\right\} .
$$

Then

$$
g(\gamma X)=\inf \left\{(|\gamma| t)^{p_{n} / H}:\left(\frac{1}{\lambda_{n}} \sum_{k \in I_{n}}\left[M\left(\frac{\bar{d}\left(X_{k}, \overline{0}\right)}{t}\right)\right]^{p_{k}}\right)^{1 / M} \leq 1, \quad n \geq 1\right\},
$$

where $t=\frac{\rho}{|\gamma|}$. Since $|\gamma|^{p_{k}} \leq \max \left(1,|\gamma|^{H}\right)$, we have

$$
\begin{aligned}
& g(\gamma X) \leq\left(\max \left(1,|\gamma|^{H}\right)\right)^{1 / M} \inf \left\{t^{p_{n} / H}:\right. \\
&\left.\left(\frac{1}{\lambda_{n}} \sum_{k \in I_{n}}\left[M\left(\frac{\bar{d}\left(X_{k}, \overline{0}\right)}{t}\right)\right]^{p_{k}}\right)^{1 / M} \leq 1, n \geq 1\right\} .
\end{aligned}
$$

So, the fact that a scalar multiplication is continuous follows from the above inequality.

Theorem 3. Let $0<h=\inf _{k} p_{k} \leq \sup _{k} p_{k}=H<\infty$. For any Orlicz function $M$ which satisfies $\Delta_{2}$-condition, we have $F[\lambda, p] \subset F[M, \lambda, p]$, where

$$
F[\lambda, p]=\left\{X=\left(X_{k}\right): \lim _{n} \frac{1}{\lambda_{n}} \sum_{k \in I_{n}}\left[M\left(\frac{\bar{d}\left(X_{k}, X_{0}\right)}{\rho}\right)\right]^{p_{k}}=0\right\}
$$

for some $\rho>0$.

Proof. Let $X=\left(X_{k}\right) \in F[\lambda, p]$ so that

$$
\lim _{n} \frac{1}{\lambda_{n}} \sum_{k \in I_{n}}\left[M\left(\frac{\bar{d}\left(X_{k}, X_{0}\right)}{\rho}\right)\right]^{p_{k}}=0 \text {, for some } \rho>0 .
$$

Let $\varepsilon>0$ and choose $\delta$ with $0<\delta<1$ such that $M(t)<\varepsilon$ for $0 \leq t \leq \delta$. Denote $y_{k}=\frac{\bar{d}\left(X_{k}, X_{0}\right)}{\rho}$ and consider

$$
\frac{1}{\lambda_{n}} \sum_{k \in I_{n}}\left[M\left(y_{k}\right)\right]^{p_{k}}<\lambda_{n} \max \left(\varepsilon, \varepsilon^{h}\right)
$$

by using continuity of $M$. For the second summation, we will make the following procedure. We have

$$
y_{k} \leq \frac{y_{k}}{\delta}<1+\frac{y_{k}}{\delta}
$$

Since $M$ is non-decreasing and convex, it follows that 


$$
M\left(y_{k}\right)<M\left(1+\frac{y_{k}}{\delta}\right) \leq \frac{1}{2} M(2)+\frac{1}{2} M\left(\frac{2 y_{k}}{\delta}\right)
$$

Since $M$ satisfies $\Delta_{2}$-condition, we can write

$$
M\left(y_{k}\right) \leq \frac{L}{2} \frac{y_{k}}{\delta} M(2)+\frac{L}{2} \frac{y_{k}}{\delta} M(2)=L \frac{y_{k}}{\delta} M(2) .
$$

We get the following estimates

$$
\begin{gathered}
\sum_{\substack{k \in I_{n} \\
y_{k}>\delta}}\left[M\left(y_{k}\right)\right]^{p_{k}} \leq \max \left(1,\left[L M(2) \delta^{-1}\right]^{H}\right) \lambda_{n} \frac{1}{\lambda_{n}} \sum_{k \in I_{n}}\left[\left(y_{k}\right)\right]^{p_{k}}, \\
\frac{1}{\lambda_{n}} \sum_{k \in I_{n}}\left[M\left(y_{k}\right)\right]^{p_{k}} \leq \max \left(\varepsilon, \varepsilon^{h}\right)+\max \left(1,\left[\frac{L}{\delta} M(2)\right]^{H}\right) \frac{1}{\lambda_{n}} \sum_{k \in I_{n}}\left[\left(y_{k}\right)\right]^{p_{k}} .
\end{gathered}
$$

Taking the limits $\varepsilon \rightarrow 0$ and $n \rightarrow \infty$, it follows that $X=\left(X_{k}\right) \in F[M, \lambda, p]$.

Theorem 4. Let $0 \leq p_{k} \leq q_{k}$ and $\left(\frac{q_{k}}{p_{k}}\right)$ be bounded. Then

$$
F[M, \lambda, q] \subset F[M, \lambda, p]
$$

Proof. The theorem is proved by using the same technique as in the proof of Theorem 3.3 by Murseleen and Basarir [7].

Now, we give some well-known definitions:

Definition 2. A sequence $X=\left(X_{k}\right)$ of fuzzy numbers is said to be statistically convergent to a fuzzy number $X_{0}$ if for every $\varepsilon>0$,

$$
\lim _{n} \frac{1}{n}\left|\left\{k \leq n: \bar{d}\left(X_{k}, X_{0}\right) \geq \varepsilon\right\}\right|=0 .
$$

We note that if a sequence $X=\left(X_{k}\right)$ of fuzzy numbers converges to a fuzzy number $X_{0}$, then it statistically converges to $X_{0}$. But the converse statement is not necessarily valid.

Definition 3. A sequence $X=\left(X_{k}\right)$ of fuzzy numbers is said to be $\lambda(p)$ statistically convergent or $S_{\lambda(p)}$ convergent to a fuzzy number $X_{0}$ if for every $\varepsilon>0$

$$
\lim _{n} \frac{1}{n}\left|\left\{k \in I_{n}:\left[\bar{d}\left(X_{k}, X_{0}\right)\right]^{p_{k}} \geq \varepsilon\right\}\right|=0,
$$

where the vertical bars indicate the number of elements in the enclosed set. In this case, we write

$$
S_{\lambda(p)}=\left\{X=\left(X_{k}\right): \lim _{n} \frac{1}{\lambda_{n}}\left|\left\{k \in I_{n}:\left[\bar{d}\left(X_{k}, X_{0}\right)\right]^{p_{k}} \geq \varepsilon\right\}\right|=0\right\} .
$$

In the case $p_{k}=1$ for all $k$, we obtain $\lambda$-statistically convergent sequence spaces $S_{\lambda}$, which was defined and studied by Savas [11]. 
Theorem 5. The following statements are valid:

$$
\begin{aligned}
& \text { a) } F[\lambda, p] \subset S_{\lambda(p)} \text {, } \\
& \text { b) if } X=\left(X_{k}\right) \in l_{\infty}(p) \cap S_{\lambda(p)} \text {, then } X=\left(X_{k}\right) \in F[\lambda, p] \text {, } \\
& \text { c) } l_{\infty}(p) \cap S_{\lambda(p)}=l_{\infty}(p) \cap F[\lambda, p],
\end{aligned}
$$

where $l_{\infty}(p)=\left\{X=\left(X_{k}\right): \sup _{k}\left[\bar{d}\left(X_{k}, X_{0}\right)\right]^{p_{k}} \leq K, K>0\right\}$.

Proof.

a) Let $\varepsilon>0$ and $X=\left(X_{k}\right) \in F[\lambda, p]$. Then we have

$$
\sum_{k \in I_{n}}\left[\bar{d}\left(X_{k}, X_{0}\right)\right]^{p_{k}} \geq \varepsilon^{H}\left|\left\{k \in I_{n}:\left[\bar{d}\left(X_{k}, X_{0}\right)\right]^{p_{k}} \geq \varepsilon\right\}\right| .
$$

Hence $X=\left(X_{k}\right) \in S_{\lambda(p)}$.

b) Suppose that $X=\left(X_{k}\right) \in S_{\lambda(p)}$ and $X=\left(X_{k}\right) \in l_{\infty}(p)$. Since $X=$ $\left(X_{k}\right)$ is bounded, we write $\left[\bar{d}\left(X_{k}, X_{0}\right)\right]^{p_{k}} \leq T$ for all $k$. Given $\varepsilon>0$, let

$$
\begin{aligned}
& A_{n}=\left|\left\{k \in I_{n}:\left[\bar{d}\left(X_{k}, X_{0}\right)\right]^{p_{k}} \geq \varepsilon\right\}\right|, \\
& B_{n}=\left|\left\{k \in I_{n}:\left[\bar{d}\left(X_{k}, X_{0}\right)\right]^{p_{k}}<\varepsilon\right\}\right| .
\end{aligned}
$$

Then we have

$$
\begin{aligned}
\frac{1}{\lambda_{n}} \sum_{k \in I_{n}}\left[\bar{d}\left(X_{k}, X_{0}\right)\right]^{p_{k}} & =\frac{1}{\lambda_{n}} \sum_{k \in A_{n}}\left[\bar{d}\left(X_{k}, X_{0}\right)\right]^{p_{k}}+\frac{1}{\lambda_{n}} \sum_{k \in B_{n}}\left[\bar{d}\left(X_{k}, X_{0}\right)\right]^{p_{k}} \\
& \leq \frac{T}{\lambda_{n}}\left|A_{n}\right|+\varepsilon^{H} .
\end{aligned}
$$

Hence $X=\left(X_{k}\right) \in F[\lambda, p]$.

c) This proof follows from (a) and (b).

Theorem 6. If $\liminf \operatorname{in}_{n} \frac{\lambda_{n}}{n}>0$, then $S_{(p)} \subset S_{\lambda(p)}$, where

$$
S_{(p)}=\left\{X=\left(X_{k}\right): \lim _{n} \frac{1}{n}\left|\left\{k \in I_{n}:\left[\bar{d}\left(X_{k}, X_{0}\right)\right]^{p_{k}} \geq \varepsilon\right\}\right|=0\right\} .
$$

Proof. Let $X=\left(X_{k}\right) \in S_{(p)}$. For given $\varepsilon>0$, we get

$$
\left\{k \leq n:\left[\bar{d}\left(X_{k}, X_{0}\right)\right]^{p_{k}} \geq \varepsilon\right\} \supset A_{n},
$$

where $A_{n}$ is the same as in Theorem 5 . Thus,

$$
\frac{1}{n}\left|\left\{k \leq I_{n}:\left[\bar{d}\left(X_{k}, X_{0}\right)\right]^{p_{k}} \geq \varepsilon\right\}\right| \geq \frac{1}{n}\left|A_{n}\right|=\frac{\lambda_{n}}{n} \frac{1}{\lambda_{n}}\left|A_{n}\right| .
$$

Taking limit as $n \rightarrow \infty$ and using $\lim \inf _{n} \frac{\lambda_{n}}{n}>0$, we get $X=\left(X_{k}\right) \in S_{\lambda(p)}$. 


\section{Acknowledgements}

The author wishes to thank Professor Binod Chandra Tripathy, Mathematical Sciences Division, Institute of Advanced Study in Science and Technology, Paschim Boragaon, Garchuk, Guwahati-781-035 India, for his kind help and friendship and also author likes to express his indebtedness to the referee for his/her comments and suggestions which improved the paper.

\section{References}

[1] P. Diamond and Kloeden P. Metric spaces of fuzzy sets. Fuzzy Sets and Systems, 35, 241-249, 1990.

[2] A. Esi. Some new sequence spaces defined by Orlicz functions. Bulletin of The Institute of Mathematics, Academia Sinica, 27(1), 71-76, 1999.

[3] A. Esi and M. Et. Some new sequence spaces defined by a sequence of Orlicz functions. Indian J. pure appl. Math., 31(8), 967-972, 2000.

[4] A. Esi, M. Isık and A. Esi. On some new sequence spaces defined by Orlicz functions. Indian J. pure appl.Math., 35(1), 31-36, 2004.

[5] J. Lindenstrauss and L. Tzafriri. On Orlicz sequence spaces. Israel J. Math., 10(3), 379-390, 1971.

[6] M. Matloka. Sequences of fuzzy numbers. BUSEFAL, 28, 28-37, 1986.

[7] Mursaleen and M. Basarir. On some new sequence spaces of fuzzy numbers. Indian J. pure appl. Math., 34(9), 1351-1357, 2003.

[8] S. Nanda. On sequences of fuzzy numbers. Fuzzy Sets and Systems, 33, 123$126,1989$.

[9] F. Nuray and E. Savas. Statistical convergence of sequences of fuzzy numbers. Math. Slovaca, 45, 269-273, 1995.

[10] S.D. Parashar and B. Choundhary. Sequence spaces defined by Orlicz functions. Indian J. pure appl. Math., 25(14), 419-428, 1994.

[11] E. Savas. On strongly $\lambda$-summable sequences of fuzzy numbers. Information Sciences, 125, 181-186, 2000.

[12] L.A. Zadeh. Fuzzy sets. Inform Control, 8, 338-353, 1965. 